\title{
GUILLAIN-BARRÉ SYNDROME AS A MANIFESTATION OF SYSTEMIC LUPUS ERYTHEMATOSUS
}

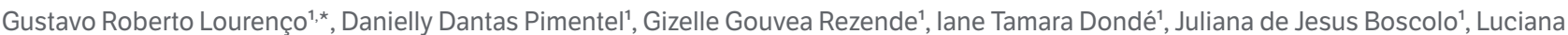
Akita', Viviane Alves Costa', Viviane Queiroz Maia', Taisa Morete Silva'

1.Faculdade de Medicina de São José do Rio Preto, São José do Rio Preto (SP), Brazil.

*Corresponding author: grlourenco@icloud.com

\section{BACKGROUND}

Systemic lupus erythematosus (SLE) is an autoimmune disease characterized by loss of self-tolerance and production of autoantibodies, which can cause manifestations in several organs and systems. About 10-15\% of cases have peripheral neurological manifestations, such as Guillain-Barré syndrome (GBS), which may precede or succeed the diagnosis of SLE.

\section{CASE REPORT}

A 26-year-old female patient presented with a 1-week history of pain and paresthesia in her lower limbs together with cyanotic toes. After one day, she developed progressive muscle weakness, which led to an inability to walk. She reported that, two months earlier, she started to have alopecia, recurrent oral ulceration and arthritis. On physical examination, she was found to have malar rash, pericardial friction rub on auscultation, toe necrosis and grade 2 muscle strength in lower limbs, along with areflexia. Complementary exams showed bilateral pleural effusion and pericardial effusion. She had an antinuclear antibody (ANA) titer of 1:1280 with a homogeneous pattern, presence of anti-dsDNA, anti-Sm, anti-Ro and p-ANCA of 1:160 and low complement levels (C3 =66 mg/dL and $\mathrm{C} 4=9 \mathrm{mg} / \mathrm{dL}$ ), which contributed to the diagnosis of SLE. Lumbar puncture was performed, showing glucose level of $27 \mathrm{mg} / \mathrm{dL}$, protein level of $51 \mathrm{mg} / \mathrm{dL}$ and WBC count of 3 cells $/ \mathrm{mm} 3$, diagnosing GBS. Pulse therapy with methylprednisolone $1 \mathrm{~g} /$ day for five consecutive days was proposed. Her paresis progressed to the rest of the body, then human immunoglobulin was administered for five days. The patient developed hemodynamic instability due to sepsis, which later caused her death despite the proposed therapy.

\section{CONCLUSION}

Guillain-Barré syndrome along with SLE is rare association. The evidence of alopecia, arthritis, malar rash, serositis and vasculitis contributed to the immunological investigation and diagnosis of SLE. The Systemic Lupus International Collaborating Clinics (SLICC - 2012) includes GBS among the neurological criteria for diagnosing SLE, making it an important aspect to be considered in the clinical decision-making process, especially when it comes to a young female patient. 